\title{
LEVANTAMENTO NUTRICIONAL DE BANANAIS DO VALE DO RIBEIRA-SP PELA ANÁLISE FOLIAR
}

\section{NUTRITIONAL SURVEY OF BANANA CROPS OF THE “VALE DO RIBEIRA-SP” BY THE FOLIAR ANALYSIS}

\author{
Leandro José Grava de GODOY ${ }^{1}$ \\ Juliana Macedo GOMES ${ }^{2}$ \\ José Carlos de MENDONÇA ${ }^{3}$
}

\begin{abstract}
RESUMO
A região do Vale do Ribeira é responsável por 70 \% da produção de banana do Estado de São Paulo. O cultivo da bananeira requer grandes quantidades balanceadas de nutrientes para um desenvolvimento adequado. $O$ levantamento dos teores foliares de nutrientes das culturas tem a finalidade de identificar a existência de deficiências e excessos de nutrientes, e direcionar as pesquisas regionais de adubação a serem planejadas. Objetivou-se com o trabalho realizar o levantamento do estado nutricional de bananais do Vale do Ribeira, SP, a partir da análise química foliar dos subgrupos Cavendish e Prata. As amostras foram coletadas em talhões representativos, no período de 1998 a 2005, nos municípios de Cajati, Eldorado, Jacupiranga, Pariquera-Açu, Registro e Sete Barras. Determinou-se os teores de N, P, K, Ca, Mg, S, B, Cu, Fe, Mn e Zn. A maioria dos bananais amostrados apresentou deficiência de $\mathrm{N}$ e S. A deficiência de $\mathrm{K}$ observada em alguns bananais deve ser devido ao desequilíbrio catiônico (K:Mg:Ca). O B foi o nutriente encontrado em excesso com maior freqüência. A média do teor foliar de K, Ca, S, Cu e Zn foi maior no subgrupo Cavendish.
\end{abstract}

Palavras-chave: Banana; análise química foliar; Musa sp.

\begin{abstract}
The region of the "Vale do Ribeira, SP" is responsible for $70 \%$ of banana production in the state of São Paulo. The cultivation of bananas plants requires large amounts of nutrients for proper development. The survey of the leaf nutrient concentration of crops aims to identify the nutrients deficiencies and excesses, and to direct regional fertilization researches to be planned. The objective of the work was to survey the nutritional status of banana crop in "Vale do Ribeira, SP". We used results of nutrient leaf analysis of and "Prata" and "Cavendish" subgroups cultivars of samples collected in banana crops representatives in "Vale do Ribeira", in the period 1998 to 2005. The leaf samples were collected in banana crops of the following counties: Cajati, Eldorado, Jacupiranga, Pariquera-Acu, Registro and Sete Barras. Were determined the leaf concentration of $\mathrm{N}, \mathrm{P}, \mathrm{K}, \mathrm{Ca}, \mathrm{Mg}$, $\mathrm{S}, \mathrm{B}, \mathrm{Cu}, \mathrm{Fe}, \mathrm{Mn}$ and $\mathrm{Zn}$. Most banana crops sampled showed deficiency of $\mathrm{N}$ and $\mathrm{S}$. The deficiency of $\mathrm{K}$ observed in some banana crops must be due to the imbalance cation $(\mathrm{K}: \mathrm{Mg}: \mathrm{Ca})$. The $\mathrm{B}$ was found in the nutrient excess more frequently. The average leaf content of $\mathrm{K}, \mathrm{Ca}, \mathrm{S}, \mathrm{Cu}$ and $\mathrm{Zn}$ was higher in "Prata" subgroup than in the "Cavendish" subgroup.
\end{abstract}

Key words: Banana; foliar analysis; Musa sp.

\footnotetext{
1 Professor Assistente Doutor do Campus Experimental de Registro, Universidade Estadual Paulista, Rua Nelson Brihi Badur, Vila Tupy, 430, 11900-000, Registro, SP. E-mail: legodoy@fregistro.unesp.br. Autor correspondente.

${ }^{2}$ Graduanda do Campus Experimental de Registro, Universidade Estadual Paulista, Registro, SP. E-mail: jumacego@gmail.com.

${ }^{3}$ Engenheiro Agrônomo, Especialista em Nutrição de Banana, Comtécnica Agropecuária, Registro, SP. E-mail: mendoncajc@uol.com.br
} 
GODOY, L.J.G. et al. Levantamento nutricional de bananais...

\section{INTRODUÇÃO}

A bananeira é uma das frutíferas mais cultivadas no Brasil, sendo importante fonte de alimentação, fixadora de mão-de-obra no meio rural e geradora de divisas para o país (Souza \& Torres Filho, 1999). As regiões que mais se destacam na produção de bananas no país são o Nordeste e o Sudeste. No sudeste, o Vale do Ribeira, SP, é importante região produtora da fruta, com área de, aproximadamente, 42,2 mil hectares e produtividade média de 22,9 t ha $^{-1}$ (2001-2006) (Gonçalves et al., 2006).

Segundo Silva \& Carvalho (2005), dentre os vários fatores ligados ao sistema de produção, a nutrição é de fundamental importância, pois, para alcançar alta produtividade econômica é necessário que a bananeira esteja equilibrada nutricionalmente.

O diagnóstico nutricional constitui-se num instrumento eficiente para detectar desequilíbrios e auxiliar no processo de recomendação de fertilizantes para bananeiras. A análise química do tecido foliar é uma ferramenta importante para avaliar o estado nutricional das plantas, em complemento à análise química do solo e à diagnose visual, e refle- te a dinâmica de nutrientes no sistema solo planta (Donato et al., 2010). Usualmente, os diagnósticos são feitos a partir da análise química do tecido foliar e posterior comparação com teores foliares ótimos (Teixeira et al., 2007).

O levantamento dos teores foliares de nutrientes das culturas tem a finalidade de identificar a existência de deficiências e excessos de nutrientes, e para direcionar as pesquisas regionais de adubação a serem planejadas (Silva \& Rodrigues, 2001).

Objetivou-se com o trabalho realizar o levantamento do estado nutricional de bananais do Vale do Ribeira, SP, avaliando dois subgrupos (Prata e Cavendish), a partir dos resultados das amostras foliares coletadas entre 1998 e 2005.

\section{MATERIAL E MÉTODOS}

Foram utilizados 1.639 resultados de análise química foliar. As amostras foram coletadas no período de 1998 a 2005, em talhões representativos de bananais dos subgrupos Prata e Cavendish, de produtores assistidos pela Comtécnica Agropecuária (Registro-SP), em seis municípios do Vale do Ribeira-SP (Tabela 1).

TABELA 1 - Número de amostras de tecidos foliares coletadas em bananais do Vale do Ribeira-SP, no período de 1998-2005, em função do município e do subgrupo da bananeira.

\begin{tabular}{cccc}
\hline Município & Subgrupo Prata & \multicolumn{2}{c}{ Subgrupo Cavendish } \\
\cline { 2 - 4 } & Prata-Anã & 8 & Nanica \\
\hline Cajati & 126 & 116 & 78 \\
Eldorado & 122 & 8 & 41 \\
Jacupiranga & 80 & 0 & 248 \\
Pariquera-Açu & 111 & 15 & 54 \\
Registro & 179 & 10 & 202 \\
Sete Barras & 127 & 157 & 114 \\
Total de amostras & 745 & & 737 \\
\hline
\end{tabular}

A amostragem foliar foi realizada segundo o método MEIR (Método de amostragem internacional de referência), citado por Rodrigues et al. (2007), o qual usa a terceira folha verdadeira a contar do ápice de plantas que estejam iniciando a emissão do cacho. Desta folha foi retirado de 10 a 15 $\mathrm{cm}$ da parte interna mediana do limbo, eliminandose a nervura central. Nessas amostras de folhas foram determinados os teores de nutrientes de acordo com métodos descritos por Malavolta et al. (2007). 
As análises estatísticas constaram da avaliação do teor médio, mínimo e máximo dos nutrientes $\mathrm{N}, \mathrm{P}, \mathrm{K}, \mathrm{Ca}, \mathrm{Mg}, \mathrm{S}, \mathrm{B}, \mathrm{Cu}, \mathrm{Fe}, \mathrm{Mn}$ e Zn das amostras foliares, coeficiente de variação e intervalo de confiança $(P<0,05)$. Os teores foliares de nutrientes foram submetidos ao teste estatístico $T$ (Bonferroni) a 5\%, conforme Furtado et al. (2008), adotando as médias de cada município como repetições, visando verificar as diferenças entre os sub- grupos Prata e Cavendish, utilizando o programa Sisvar v. 4.2 (Ferreira, 2000). Realizou-se o estudo de freqüência de ocorrência de bananais com teores deficientes, adequados e altos, tomando-se por base os teores para a cultura da bananeira (Tabela 2). A porcentagem de $\mathrm{Ca}, \mathrm{Mg}$ e $\mathrm{K}$ em relação ao total dos cátions na folha e as relações $\mathrm{Ca} / \mathrm{Mg}, \mathrm{K} /$ Ca e K/Mg foram calculadas segundo López \& Espinosa (1995).

TABELA 2 - Limites de interpretação dos macronutrientes e micronutrientes da análise química foliar.

\begin{tabular}{|c|c|c|c|c|c|c|c|c|c|c|c|}
\hline \multirow[t]{3}{*}{ Classificação } & \multicolumn{11}{|c|}{ Nutrientes } \\
\hline & $\mathrm{N}^{(1}$ & $P^{(1)}$ & $\mathrm{K}^{(1)}$ & $\mathrm{Ca}^{(1)}$ & $\underset{(1)}{\mathrm{Mg}}$ & $S^{(1)}$ & $\mathrm{Zn}^{(1)}$ & $\mathrm{Fe}^{(1)}$ & $\mathrm{Mn}^{(1)}$ & $\mathrm{Cu}^{(1)}$ & 1) $\mathrm{B}^{(2)}$ \\
\hline & \multicolumn{11}{|c|}{ - } \\
\hline Deficiente & $<27$ & $<1,8$ & $\begin{array}{l}<35 \\
(2)\end{array}$ & $<3$ & $<3$ & $<2,5$ & $<20$ & $<80$ & $<200$ & $<6$ & $<10$ \\
\hline Adequado & $27-36$ & $\begin{array}{l}1,8- \\
2,7\end{array}$ & $\begin{array}{l}30- \\
54\end{array}$ & $3-12$ & $3-6$ & $2,5-8$ & $20-50$ & $\begin{array}{l}80- \\
360\end{array}$ & $\begin{array}{l}200- \\
2000\end{array}$ & $6-30$ & $\begin{array}{l}10- \\
25\end{array}$ \\
\hline Excesso & $>36$ & $>2,7$ & $>54$ & $>12$ & $>6$ & $>8$ & $>50$ & $>360$ & $>2000$ & $>30$ & $>25$ \\
\hline
\end{tabular}

${ }^{(1)}$ Raij et al.(1997); (2) foi considerado o teor de $30 \mathrm{~g} \mathrm{~kg}^{-1} \mathrm{~K}$ para o subgrupo Prata, segundo Silva \& Rodrigues (2001).

\section{RESULTADOS E DISCUSSÃO}

Dos bananais amostrados nos municípios de Registro, Eldorado e Cajati (Figuras 1A, 1D e $2 \mathrm{~A})$, mais de $70 \%$ apresentaram teores deficientes de $\mathrm{N}$ na folha $\left(<27 \mathrm{~g} \mathrm{~kg}^{-1}\right)$ independente do subgrupo (Prata ou Cavendish). Nos demais municípios, a deficiência de $\mathrm{N}$ foi constatada em 59 a $74,8 \%$ dos bananais, dependendo do subgrupo. A mais alta frequência da deficiência de $\mathrm{N}$ ocorreu nos talhões de Cajati, no subgrupo Cavendish $(80,2 \%)$ (Figura $2 \mathrm{~A})$. As médias dos teores de $\mathrm{N}$ na folha, para ambos os subgrupos, variaram de 24 a $26 \mathrm{~g} \mathrm{~kg}^{-1}$, considerado deficiente (Tabelas 2, 3 e 4). A alta frequência da deficiência de $\mathrm{N}$ pode ser explicada pela grande quantidade de chuva na região e menor parcelamento da adubação, favorecendo perdas por lixiviação N (Carpena et al., 2002; Fey et al., 2010); por volatilização (Prasertak et al., 2001) e também ao uso de doses reduzidas de N.A maioria (mais de $62 \%$ ) dos bananais do subgrupo Cavendish apresentou teor foliar adequado de P. Segundo Godoy et al. (2009) a adubação fosfatada de cobertura é frequentemente utilizada na região, empregando-se fertilizantes mistos (NPK), independente do teor de $\mathrm{P}$ no solo, levando a teores médios e altos de $\mathrm{P}$ no solo. Além disso, segundo Soares et al. (2008), a demanda por $\mathrm{P}$ pela bananeira é baixa, devido ser móvel no floema e pouco exportado pelo cacho (15 $\mathrm{kg} \mathrm{ha}^{-1} \mathrm{P}$ ) sendo difícil de observar sintomas da deficiência no campo. As mais altas frequências da deficiência de $P$ foram observadas nos bananais do subgrupo Prata dos municípios de Registro (40,8\%), Eldorado $(36,9 \%)$ e Cajati $(39,7 \%)$ provavelmente devido à presença de solos mais argilosos, onde a eficiência da adubação fosfatada é mais baixa. A média do teor de $P$, para os dois subgrupos variou de 1,9 a $2,2 \mathrm{~g} \mathrm{~kg}^{-1}$, considerado adequado (Tabelas 2, 3 e 4).

Para os bananais do subgrupo Cavendish, mais de $40 \%$ dos talhões amostrados apresentou teor de $\mathrm{K}$ adequado nas folhas (35 a $54 \mathrm{~g} \mathrm{~kg}^{-1}$ ) (Figuras 1C, 1F, 2C, 2F, 3C e 3F). A deficiência de $\mathrm{K}$ foi mais frequente nos bananais do subgrupo Prata, principalmente nos municípios de Eldorado, Jacupiranga e Pariquera-Açu (Figura 1D, 2D e 3D). Esta carência pode estar relacionada com o equilíbrio catiônico, pois, nos bananais destes municípios, verifica-se a menor porcentagem de $\mathrm{K}$ e as maiores de $\mathrm{Ca}$ e $\mathrm{Mg}$ na folha (Tabela 5), em relação aos demais. A porcentagem de $\mathrm{K}$ na folha está abaixo (55 a $61 \%$ de $\mathrm{K}$ ) e a de $\mathrm{Mg}$ acima (18 a $20 \%$ de $\mathrm{Mg}$ ) da estabelecida por López \& Espinosa (1995) para o equilíbrio catiônico mais adequado. Segundo Godoy et al. (2009) os teores de Mg encontrados nos bananais do Vale do Ribeira são altos devido ao uso frequente de calcário dolomítico. A média do teor foliar de $\mathrm{K}$ para o subgrupo Prata foi de $31 \mathrm{~g} \mathrm{~kg}^{-1}$, menor que a média para o subgrupo Cavendish, de $34 \mathrm{~g} \mathrm{~kg}^{-1}$ (Tabela 6), corroborando com Damatto Junior et al. (2006), que afirmam que os teores foliares adequados de $\mathrm{K}$ nas bananeiras do subgrupo Prata são menores que nas do subgrupo Cavendish. 
GODOY, L.J.G. et al. Levantamento nutricional de bananais...
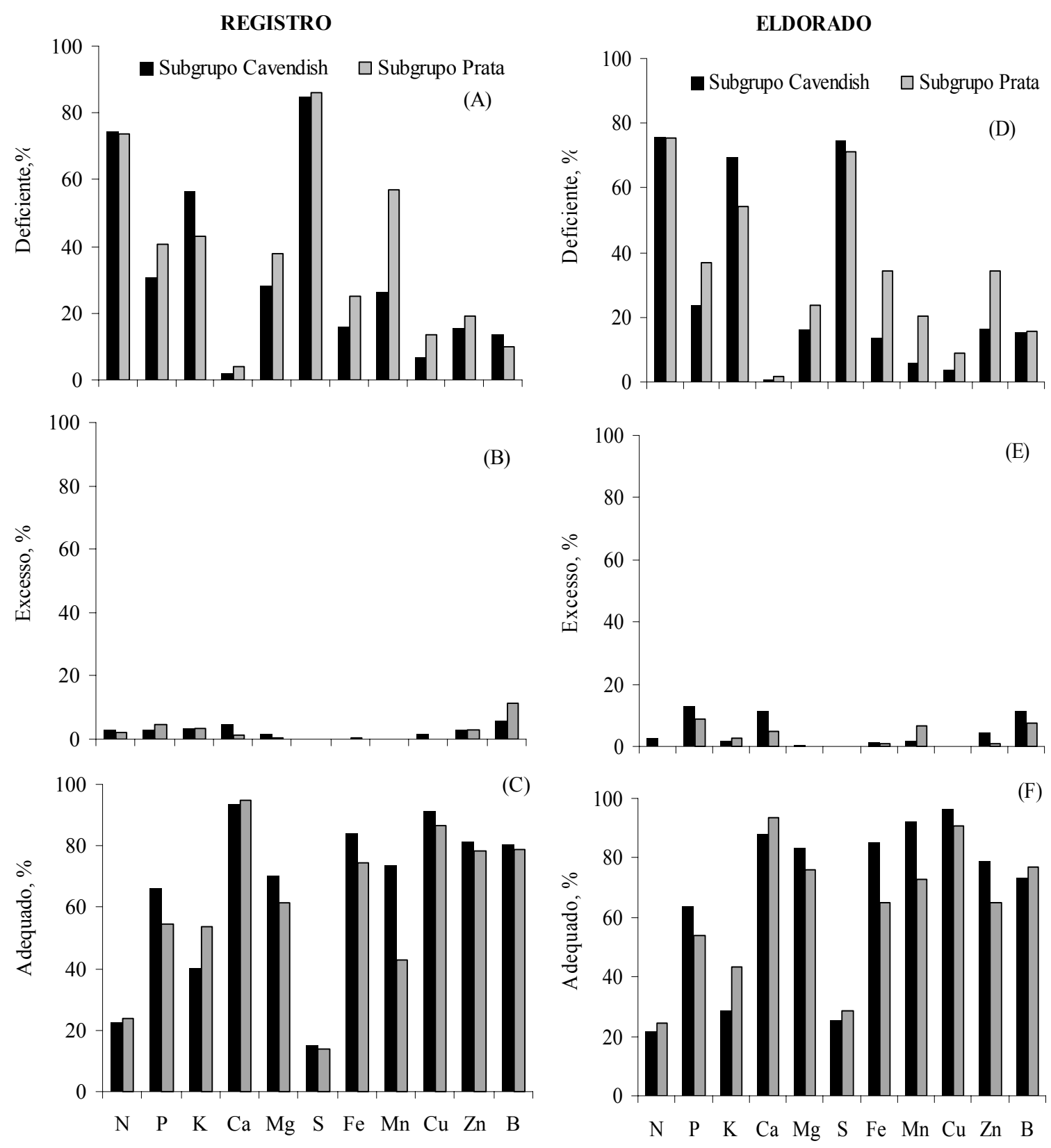

FIGURA 1 - Frequência de talhões de bananais dos subgrupos Cavendish e Prata, nos municípios de Registro ( $\mathrm{A}, \mathrm{B}$ e $\mathrm{C}$ ) e de Eldorado ( $\mathrm{D}, \mathrm{E}$ e $\mathrm{F}$ ), com deficiência, excesso e teores adequados dos nutrientes das análises foliares (1998 a 2005). 
GODOY, L.J.G. et al. Levantamento nutricional de bananais...
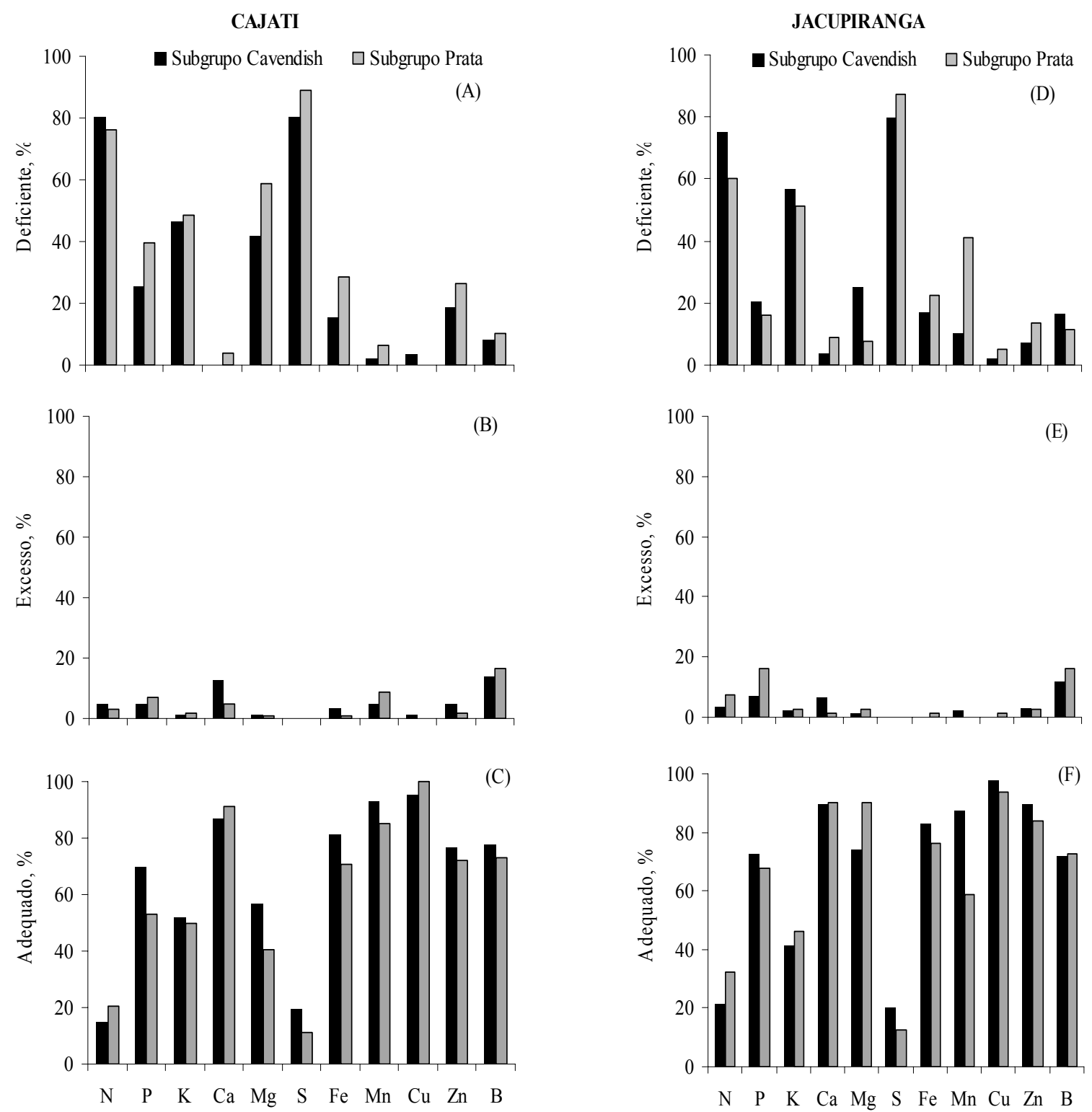

FIGURA 2 - Frequência de talhões de bananais dos subgrupos Cavendish e Prata, nos municípios de Cajati (A, $B$ e C) e Jacupiranga (D, E e F) com deficiência, excesso e teores adequados dos nutrientes das análises foliares (1998 a 2005). 
GODOY, L.J.G. et al. Levantamento nutricional de bananais...

SETE BARRAS
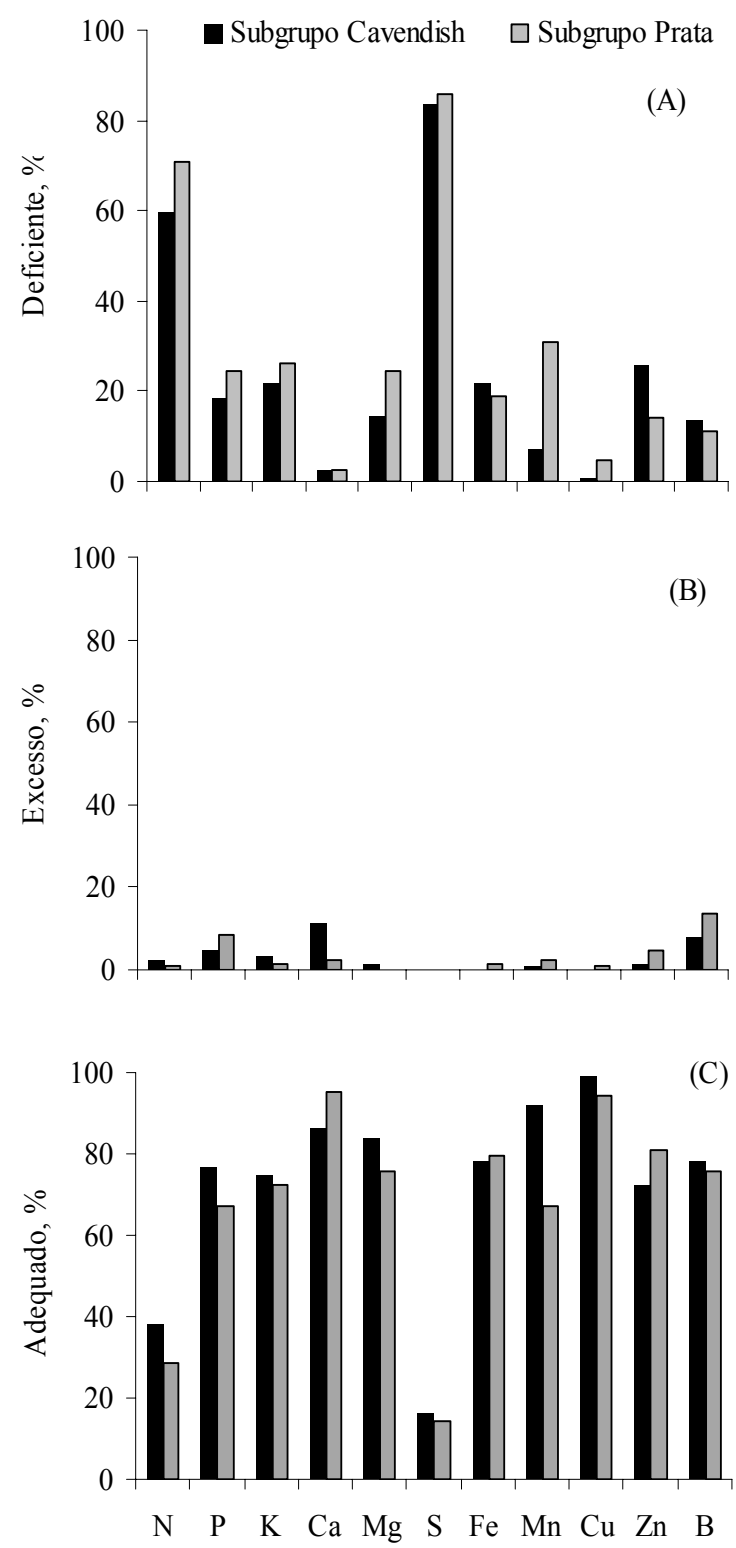

PARIQUERA-AÇU
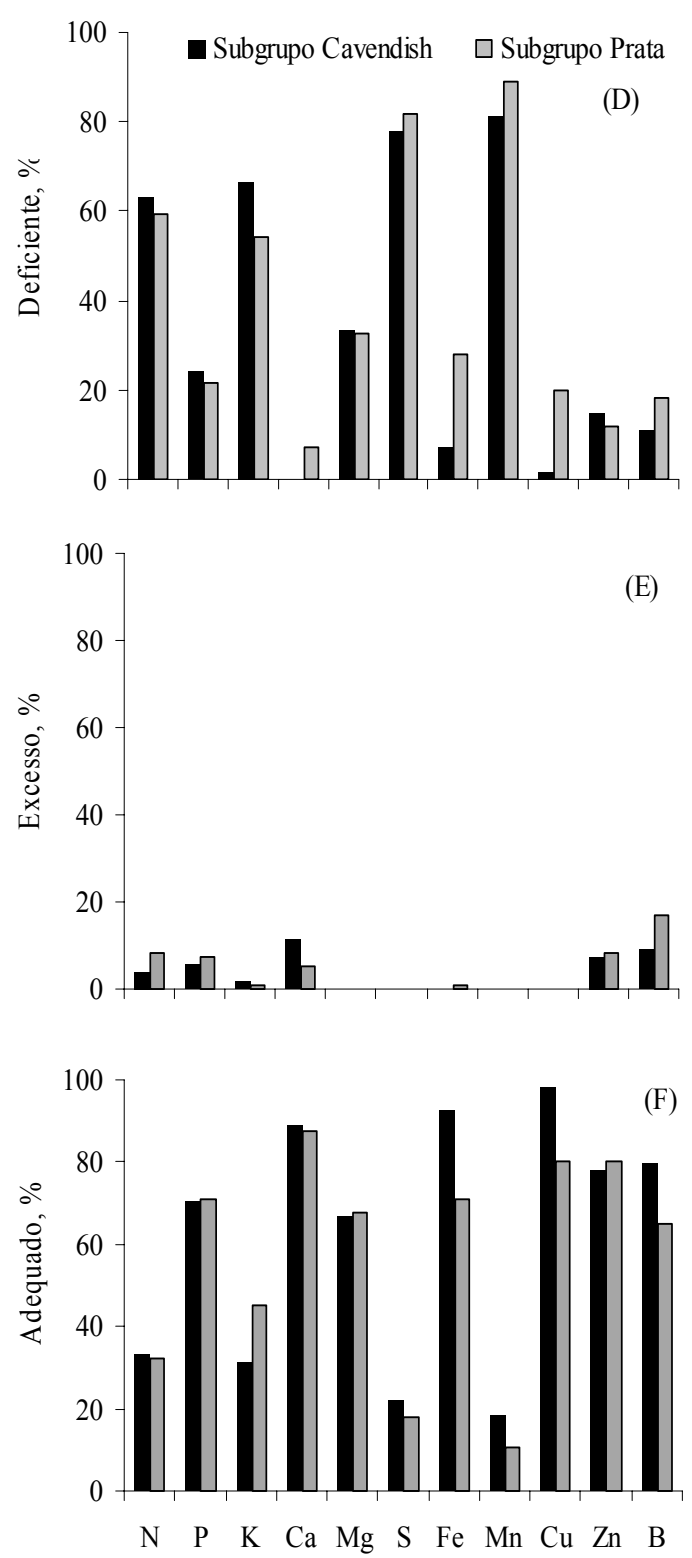

FIGURA 3 - Frequência de talhões de bananais dos subgrupos Cavendish e Prata, nos municípios de Sete Barras ( $A, B$ e C) e Pariquera-Açu (D, E e F), com deficiência, excesso e teores adequados dos nutrientes das análises foliares (1998 a 2005). 
GODOY, L.J.G. et al. Levantamento nutricional de bananais...

TABELA 3 - Valores médios, máximos e mínimos, coeficiente variação e intervalos de confiança dos teores da terceira folha em 894 amostras coletadas em bananais do subgrupo Cavendish nos municípios do Vale do Ribeira, SP, no período de 1998 a 2005.

\begin{tabular}{|c|c|c|c|c|c|c|c|c|c|c|c|}
\hline Medidas & $\mathrm{N}$ & $P$ & $\mathrm{~K}$ & $\mathrm{Ca}$ & Mg & $S$ & Fe & Mn & $\begin{array}{c}\mathrm{Cu} \\
\mathrm{g}^{-1}----\end{array}$ & $\mathrm{Zn}$ & $B$ \\
\hline \multicolumn{12}{|c|}{ Cajati } \\
\hline Mín. & 12 & 1,0 & 4 & 3 & 1 & 1,0 & 52 & 60 & 5 & 10 & 7 \\
\hline Máx. & 40 & 4,0 & 55 & 26 & 6 & 4,0 & 950 & 2800 & 205 & 62 & 46 \\
\hline Méd. & 24 & 2 & 36 & 9 & 3 & 2,0 & 132 & 967 & 13 & 30 & 18 \\
\hline CV, \% & 23,0 & 19,6 & 26,4 & 42,2 & 29,7 & 31,0 & 80,1 & 58,0 & 157,0 & 35,7 & 42,7 \\
\hline $\begin{array}{c}\text { IC } \\
(P<0,05)\end{array}$ & $22-25$ & $2,0-2,2$ & $34-38$ & $8-10$ & $3,0-3,4$ & $1,9-2,2$ & $110-155$ & $849-1085$ & $9-18$ & $28-32$ & $16-19$ \\
\hline \multicolumn{12}{|c|}{ Eldorado } \\
\hline Mín. & 13 & 1,0 & 5 & 2 & 1 & 0,9 & 53 & 65 & 3 & 7 & 5 \\
\hline Máx. & 51 & 5,6 & 74 & 20 & 6 & 3,9 & 430 & 2380 & 27 & 70 & 55 \\
\hline Méd. & 24 & 2,2 & 31 & 9 & 4 & 2,2 & 117 & 741 & 10 & 28 & 17 \\
\hline $\mathrm{CV}, \%$ & 23,6 & 36,8 & 30,5 & 34,6 & 22,9 & 25,0 & 43,7 & 64,9 & 34,7 & 38,8 & 48,7 \\
\hline $\begin{array}{c}I C \\
(P<0,05)\end{array}$ & $23-25$ & $2,1-2,4$ & $30-33$ & $8-9$ & $3,7-3,9$ & $2,1-2,3$ & $109-125$ & $666-817$ & $10-11$ & $26-30$ & $16-18$ \\
\hline \multicolumn{12}{|c|}{ Jacupiranga } \\
\hline Mín. & 9 & 1,0 & 13 & 1 & 1 & 0,6 & 50 & 20 & 3 & 11 & 2 \\
\hline Máx. & 47 & 4,4 & 64 & 24 & 8 & 5,2 & 300 & 3500 & 26 & 995 & 165 \\
\hline Méd. & 24 & 2,1 & 34 & 8 & 3 & 2,1 & 113 & 700 & 10 & 35 & 18 \\
\hline $\mathrm{CV}, \%$ & 22,7 & 22,3 & 26,1 & 38,7 & 25,2 & 29,7 & 37,6 & 68,5 & 31,7 & 181,4 & 79,6 \\
\hline $\begin{array}{c}\mathrm{IC} \\
(\mathrm{P}<0,05)\end{array}$ & $24-25$ & $2,0-2,1$ & 33-35 & $7-8$ & $3,3-3,6$ & $2,0-2,2$ & $108-118$ & $642-759$ & $10-11$ & $27-43$ & $16-19$ \\
\hline \multicolumn{12}{|c|}{ Pariquera-Açu } \\
\hline Mín. & 15 & 1,1 & 10 & 4 & 1 & 1,3 & 55 & 10 & 5 & 11 & 6 \\
\hline Máx. & 40 & 4,3 & 56 & 19 & 5 & 3,2 & 260 & 400 & 18 & 82 & 57 \\
\hline Méd. & 25 & 2,1 & 32 & 9 & 3 & 2,1 & 128 & 137 & 11 & 31 & 17 \\
\hline $\mathrm{CV}, \%$ & 19,6 & 25,2 & 29,9 & 31,6 & 20,8 & 21,0 & 34,8 & 63,0 & 28,5 & 47,5 & 47,2 \\
\hline $\begin{array}{c}\mathrm{IC} \\
(\mathrm{P}<0,05)\end{array}$ & $24-27$ & $1,9-2,2$ & $30-35$ & $8-9$ & $3,0-3,4$ & $2,0-2,3$ & $115-139$ & $114-160$ & $10-12$ & $27-34$ & $14-19$ \\
\hline \multicolumn{12}{|c|}{ Registro } \\
\hline Mín. & 9 & 1,0 & 12 & 1 & 1 & 0,9 & 21 & 17 & 2 & 8 & 5 \\
\hline Máx. & 45 & 6,3 & 70 & 22 & 10 & 5,5 & 250 & 1970 & 35 & 230 & 48 \\
\hline Méd. & 24 & 2,0 & 34 & 7 & 3 & 2,0 & 111 & 424 & 11 & 29 & 16 \\
\hline CV, \% & 21,7 & 24,1 & 29,6 & 35,8 & 28,3 & 28,3 & 34,6 & 81,2 & 42,2 & 72,2 & 40,4 \\
\hline $\begin{array}{c}I C \\
(P<0,05)\end{array}$ & $23-25$ & $1,9-2,0$ & $33-36$ & $7-8$ & $3,3-3,6$ & $1,9-2,0$ & $106-116$ & $379-470$ & $10-11$ & $26-32$ & $15-17$ \\
\hline \multicolumn{12}{|c|}{ Sete Barras } \\
\hline Mín. & 15 & 1,0 & 16 & 1 & 2 & 1,0 & 42 & 80 & 4 & 6 & 5 \\
\hline Máx. & 43 & 4,3 & 70 & 16 & 6 & 3,4 & 360 & 2160 & 28 & 550 & 120 \\
\hline Méd. & 25 & 2,1 & 35 & 8 & 4 & 2,0 & 111 & 664 & 11 & 29 & 17 \\
\hline $\mathrm{CV}, \%$ & 20,3 & 19,9 & 26,4 & 34,8 & 22,6 & 23,4 & 43,3 & 53,9 & 31,1 & 165,0 & 65,6 \\
\hline $\begin{array}{c}I C \\
(P<0,05)\end{array}$ & $25-26$ & $2,0-2,1$ & $33-37$ & $8-9$ & $3,6-3,9$ & $1,9-2,1$ & $103-120$ & $601-727$ & $10-11$ & $21-37$ & $15-19$ \\
\hline
\end{tabular}

IC - intervalo de confiança; CV - coeficiente de variação. 
GODOY, L.J.G. et al. Levantamento nutricional de bananais...

TABELA 4 - Valores médios, máximos e mínimos, coeficiente variação e intervalo de confiança dos teores da terceira folha em 745 amostras coletadas em bananais do subgrupo Prata nos municípios do Vale do Ribeira, SP, no período de 1998 a 2005.

\begin{tabular}{|c|c|c|c|c|c|c|c|c|c|c|c|}
\hline Medidas & $\mathrm{N}$ & $\begin{array}{c}P \\
\end{array}$ & $\begin{array}{c}\mathrm{K} \\
-\mathrm{g} \mathrm{kg}^{-1}\end{array}$ & $\mathrm{Ca}$ & $\mathrm{Mg}$ & S & $\mathrm{Fe}$ & $\begin{array}{c}\mathrm{Mn} \\
\mathrm{y}\end{array}$ & $g^{-1} \begin{array}{c}\mathrm{Cu} \\
\mathrm{g}^{-1}\end{array}$ & $\mathrm{Zn}$ & $B$ \\
\hline \multicolumn{12}{|c|}{ Cajati } \\
\hline Mín. & 14 & 1,2 & 14 & 2 & 1 & 0,5 & 40 & 14 & 4 & 7 & 4 \\
\hline Máx. & 40 & 4,5 & 60 & 15 & 7 & 3,6 & 415 & 9500 & 23 & 68 & 133 \\
\hline Méd. & 24 & 2,0 & 31 & 7 & 3 & 2,0 & 106 & 1183 & 10 & 25 & 19 \\
\hline $\mathrm{CV}, \%$ & 19,7 & 27,0 & 30,3 & 36,6 & 33,5 & 28,1 & 48,3 & 140,5 & 33,7 & 38,5 & 69,8 \\
\hline $\mathrm{IC}(\mathrm{P}<0,05)$ & $23-25$ & $1,8-2,0$ & $29-32$ & $6-7$ & $2,8-3,1$ & $1,8-2,0$ & $97-115$ & $892-1473$ & $9-10$ & $23-27$ & $17-22$ \\
\hline \multicolumn{12}{|c|}{ Eldorado } \\
\hline Mín. & 11 & 0,7 & 8 & 2 & 1 & 0,7 & 45 & 32 & 2 & 7 & 2 \\
\hline Máx. & 35 & 3,8 & 95 & 14 & 6 & 3,7 & 380 & 3100 & 25 & 52 & 54 \\
\hline Méd. & 24 & 2,0 & 30 & 7 & 4 & 2,0 & 99 & 753 & 9 & 23 & 15 \\
\hline $\mathrm{CV}, \%$ & 19,0 & 28,0 & 38,2 & 35,8 & 25,7 & 27,1 & 44,7 & 88,7 & 39,6 & 39,3 & 47,9 \\
\hline $\mathrm{IC}(\mathrm{P}<0,05)$ & $23-25$ & $1,8-2,0$ & $28-32$ & $7-8$ & $3,5-3,8$ & $1,8-2,0$ & $91-107$ & $635-872$ & $9-10$ & $21-24$ & 14-17 \\
\hline \multicolumn{12}{|c|}{ Jacupiranga } \\
\hline Mín. & 16 & 1,2 & 9 & 1 & 2 & 0,8 & 43 & 43 & 2 & 10 & 5 \\
\hline Máx. & 17 & 5,5 & 57 & 16 & 8 & 4,1 & 490 & 1600 & 45 & 70 & 64 \\
\hline Méd. & 26 & 2,2 & 30 & 6 & 4 & 2,0 & 106 & 399 & 9 & 30 & 19 \\
\hline $\mathrm{CV}, \%$ & 21,4 & 28,4 & 27,3 & 41,4 & 24,6 & 28,6 & 52,0 & 89,3 & 49,6 & 32,3 & 47,6 \\
\hline $\mathrm{IC}(\mathrm{P}<0,05)$ & $25-27$ & $2,1-2,4$ & $28-32$ & $6-7$ & $3,6-4,1$ & $1,9-2,2$ & $94-118$ & $321-478$ & $8-10$ & $28-31$ & $17-21$ \\
\hline \multicolumn{12}{|c|}{ Pariquera-Açu } \\
\hline Mín. & 16 & 0,9 & 2 & 2 & 2 & 0,5 & 30 & 5 & 1 & 8 & 5 \\
\hline Máx. & 63 & 3,8 & 60 & 26 & 6 & 4,5 & 605 & 1200 & 25 & 75 & 54 \\
\hline Méd. & 26 & 2,1 & 29 & 7 & 3 & 2,0 & 104 & 112 & 8 & 30 & 17 \\
\hline $\mathrm{CV}, \%$ & 26,3 & 23,4 & 33,7 & 49,9 & 26,1 & 32,9 & 55,8 & 122,1 & 50,8 & 41,7 & 51,9 \\
\hline IC $(P<0,05)$ & $25-27$ & $2,0-2,2$ & $27-31$ & $6-8$ & $3,2-3,5$ & $1,9-2,2$ & 93-115 & $87-138$ & $7-9$ & $28-32$ & $16-19$ \\
\hline \multicolumn{12}{|c|}{ Registro } \\
\hline Mín. & 12 & 1,1 & 8 & 1 & 1 & 0,6 & 15 & 10 & 2 & 10 & 5 \\
\hline Máx. & 29 & 3,6 & 73 & 15 & 8 & 4,0 & 390 & 1720 & 27 & 63 & 237 \\
\hline Méd. & 24 & 1,9 & 32 & 6 & 3 & 1,8 & 101 & 283 & 9 & 28 & 19 \\
\hline $\mathrm{CV}, \%$ & 20,2 & 22,5 & 31,3 & 32,6 & 27,2 & 32,3 & 40,5 & 110,1 & 37,6 & 35,8 & 100,3 \\
\hline $\mathrm{IC}(\mathrm{P}<0,05)$ & $24-25$ & $1,8-2,0$ & $31-34$ & $6-7$ & $3,1-3,4$ & $1,8-1,9$ & $95-107$ & $237-328$ & $8-9$ & $26-29$ & $16-21$ \\
\hline \multicolumn{12}{|c|}{ Sete Barras } \\
\hline Mín. & 12 & 1,3 & 9 & 2 & 1 & 0,7 & 27 & 20 & 3 & 9 & 3 \\
\hline Máx. & 37 & 3,9 & 71 & 13 & 6 & 4,4 & 1400 & 2500 & 138 & 92 & 41 \\
\hline Méd. & 24 & 2,1 & 34 & 7 & 4 & 1,8 & 122 & 440 & 11 & 30 & 16 \\
\hline $\mathrm{CV}, \%$ & 21,8 & 23,6 & 26,6 & 31,5 & 22,4 & 31,2 & 102,2 & 99,0 & 108,7 & 42,2 & 42,5 \\
\hline IC $(P<0,05)$ & $23-25$ & $2,0-2,2$ & $32-36$ & $6-7$ & $3,4-3,7$ & $1,7-1,9$ & $100-143$ & $364-516$ & $9-13$ & $28-32$ & $15-18$ \\
\hline
\end{tabular}

IC - intervalo de confiança; CV - coeficiente de variação. 
GODOY, L.J.G. et al. Levantamento nutricional de bananais...

TABELA 5 - Relações entre cátions da terceira folha em bananais nos municípios do Vale do Ribeira, SP, no período de 1998 a 2005.

\begin{tabular}{|c|c|c|c|c|c|c|}
\hline \multirow{2}{*}{ Cidades } & $\mathrm{Ca} /(\mathrm{Ca}+\mathrm{Mg}+\mathrm{K})$ & $\mathrm{Mg} /(\mathrm{Ca}+\mathrm{Mg}+\mathrm{K})$ & $\mathrm{K} /(\mathrm{Ca}+\mathrm{Mg}+\mathrm{K})$ & $\mathrm{Ca} / \mathrm{Mg}$ & $\mathrm{K} / \mathrm{Ca}$ & $\mathrm{K} / \mathrm{Mg}$ \\
\hline & \multicolumn{5}{|c|}{ Subgrupo Prata } & \\
\hline Cajati & 25,16 & 17,78 & 57,07 & 2,36 & 4,42 & 10,43 \\
\hline Eldorado & 25,32 & 21,08 & 53,60 & 2,00 & 4,13 & 8,26 \\
\hline Jacupiranga & 22,24 & 22,88 & 54,88 & 1,62 & 4,81 & 7,79 \\
\hline Pariquera-Açu & 25,36 & 20,48 & 54,15 & 2,06 & 4,16 & 8,59 \\
\hline Registro & 21,97 & 19,18 & 58,85 & 1,91 & 5,22 & 9,97 \\
\hline Sete Barras & 22,56 & 19,79 & 57,65 & 1,90 & 4,98 & 9,47 \\
\hline \multirow[t]{2}{*}{ Média } & 23,77 & 20,20 & 56,03 & 1,98 & 4,62 & 9,09 \\
\hline & \multicolumn{5}{|c|}{ Subgrupo Cavendish } & \\
\hline Cajati & 27,03 & 16,54 & 56,43 & 2,72 & 4,07 & 11,09 \\
\hline Eldorado & 28,04 & 20,45 & 51,52 & 2,29 & 3,58 & 8,19 \\
\hline Jacupiranga & 25,07 & 18,74 & 56,20 & 2,23 & 4,37 & 9,75 \\
\hline Pariquera-Açu & 28,34 & 17,71 & 53,95 & 2,67 & 3,71 & 9,90 \\
\hline Registro & 24,19 & 18,71 & 57,10 & 2,15 & 4,60 & 9,92 \\
\hline Sete Barras & 25,43 & 19,26 & 55,31 & 2,20 & 4,24 & 9,34 \\
\hline Média & 26,35 & 18,57 & 55,08 & 2,38 & 4,10 & 9,70 \\
\hline
\end{tabular}

Os teores de Ca, para ambos os subgrupos, foram adequados ( 3 a $12 \mathrm{~g} \mathrm{~kg}^{-1}$ ) em mais de $86 \%$ dos talhões amostrados em todos os municípios (Figuras 1C, 1F, 2C, 2F, 3C e 3F). Os teores adequados podem ser justificados pelos altos teo- res de Ca presentes no solo também devido à aplicação periódica de calcário, segundo Godoy et al. (2009). Os bananais do subgrupo Prata apresentaram média inferior do teor foliar de $\mathrm{Ca}$ em relação aos bananais do subgrupo Cavendish (Tabela 6).

TABELA 6 - Médias dos teores da terceira folha em bananais dos subgrupos Prata e Cavendish nos municípios do Vale do Ribeira, SP, no período de 1998 a 2005.

\begin{tabular}{|c|c|c|c|c|c|c|c|c|c|c|c|}
\hline Medidas & $\mathrm{N}$ & $P$ & $\begin{array}{c}\mathrm{K} \\
\mathrm{kg}^{-1}\end{array}$ & $\mathrm{Ca}$ & $\mathrm{Mg}$ & $S$ & $\mathrm{Fe}$ & $\mathrm{Mn}$ & $\begin{array}{c}\mathrm{Cu} \\
\mathrm{kg}^{-1}\end{array}$ & $\mathrm{Zn}$ & $B$ \\
\hline Prata & 25 & 2,0 & $31 b^{1}$ & $7 b$ & 3,4 & $1,9 b$ & 106 & 528 & $9 b$ & $27 b$ & 17 \\
\hline Cavendish & 25 & 2,1 & $34 a$ & $8 a$ & 3,5 & $2,1 a$ & 119 & 606 & $11 a$ & $30 a$ & 18 \\
\hline
\end{tabular}

\footnotetext{
${ }^{1}$ Médias seguidas de mesma letra não diferem entre si pelo teste de Bonferroni a $5 \%$ de probabilidade.
}

A frequência da deficiência de Mg no município de Cajati foi maior que $40 \%$ (Figura 2A), para os dois subgrupos de cultivares. Esta deficiência de $\mathrm{Mg}$, provavelmente, ocorreu devido aos teores altos de $\mathrm{Ca}$ e $\mathrm{K}$, verificados pela maior relação $\mathrm{Ca} / \mathrm{Mg}$ e $\mathrm{K} / \mathrm{Mg}$ (Tabela 3 ) observada nos bananais deste município. A porcentagem de $\mathrm{Mg}$ em relação aos macronutrientes catiônicos também foi inferior nos bananais de Cajati em relação aos bananais dos demais municípios. Segundo Borges (2004) a elevada relação $\mathrm{K} / \mathrm{Mg}$ pode favorecer o aparecimento do "azul-da-bananeira", caracterizada pela coloração púrpura nos pecíolos das folhas afetadas, que se manifesta quando a relação $\mathrm{K} / \mathrm{Mg}$ (teores em $\mathrm{g}$ $\mathrm{kg}^{-1}$ ) nas folhas é maior que 13,0 no período de florescimento das plantas. Este sintoma da deficiência é observado em alguns bananais do Vale do Ribeira (Mendonça et al., 2006). A média do teor de
Mg variou de 3 a $4 \mathrm{~g} \mathrm{~kg}^{-1}$, para ambos os subgrupos de cultivares, considerado adequado.

A deficiência de $S$ foi constatada em mais de $80 \%$ dos bananais amostrados (exceto nos taIhões do subgrupo Cavendish dos municípios de Pariquera-Açu e Eldorado) (Figuras 1A, 1D, 2A, 2D, 3A e 3D) independente do subgrupo. A alta deficiência de $S$ pode ser explicada pela utilização de fertilizantes mistos mais concentrados (Mendonça et al., 2006), que não possuem S, pela alta lixiviação do sulfato e a não utilização do sulfato de amônio como fonte de $\mathrm{N}$, por ser uma fonte acidificante que favorece a ocorrência da fusariose (Mal-doPanamá) (Moraes \& Lima, 2006). A média do teor foliar de $S$ foi maior no subgrupo Prata (Tabela 6), embora em ambos os subgrupos tenha sido deficiente (Tabela 2). 
GODOY, L.J.G. et al. Levantamento nutricional de bananais...

A maior parte dos bananais apresentou teor adequado de $\mathrm{Fe}$ e $\mathrm{Mn}$ nas folhas (Figuras $1 \mathrm{C}$, $1 F, 2 C, 2 F, 3 C$ e $3 F)$. A frequência da deficiência de Fe foi maior que $30 \%$ no bananais do subgrupo Prata, principalmente em Eldorado, Cajati e Pariquera-Açu (Figuras 1D, 2A e 3A). Para os dois subgrupos, a média do teor de Fe variou de 99 a 132 $\mathrm{mg} \mathrm{kg}^{-1}$. Em relação ao $\mathrm{Mn}$, destacaram-se os bananais de Pariquera-Açu, chegando a frequência de deficiência a 89,1 e $81,4 \%$, para o subgrupo Prata e Cavendish, respectivamente (Figura $3 \mathrm{~A}$ ), em decorrência do baixo teor deste nutriente nos solos dos bananais deste município (Godoy et al., 2009). O $\mathrm{Mn}$ foi o elemento que apresentou maior variação do teor médio, corroborando com os resultados obtidos por Silva \& Rodrigues (2001), ficando na faixa 112 a $1183 \mathrm{mg} \mathrm{kg}^{-1}$, ocorrendo a menor média nos bananais de Pariquera-Açu e a maior média em Cajati, onde um dos talhões apresentou $9.500 \mathrm{mg}$ $\mathrm{kg}^{-1} \mathrm{Mn}$, com sintomas visuais de excesso (Tabela 2). Segundo Mendonça et al. (2006) é comum a observação de sintomas visuais da deficiência de Mn em bananais de Pariquera-Açu e de toxidez em Cajati.

Mais de $80 \%$ dos talhões amostrados apresentou teor adequado de $\mathrm{Cu}$ (Figuras 1C, 1F, 2C, 2F, 3C e 3F). Segundo López \& Espinosa (1995) é muito rara a deficiência de $\mathrm{Cu}$ na bananeira. Além disso, os solos de bananais do Vale do Ribeira normalmente apresentam alto teor de Cu (Godoy et al., 2009).

Em relação ao $\mathrm{Zn}$ e o $\mathrm{B}$ a maior parte dos bananais apresentou teor adequado. Os bananais do subgrupo Cavendish apresentaram média superior do teor foliar de $\mathrm{Cu}$ e $\mathrm{Zn}$ em relação aos bananais do subgrupo Prata (Tabela 6).

Dentre os nutrientes, o B é o que foi observado em excesso com maior freqüência nos dois subgrupos de cultivares (Figuras 1B, 1E, 2B, 2E, 3B e $3 E$ ), chegando a $17,1 \%$ dos bananais de Pariquera-Açu. A aplicação desuniforme das fontes de $B$ via solo pode ser uma das causas do excesso deste elemento em alguns bananais (Vargas et al., 2007).

\section{CONCLUSÕES}

No Vale do Ribeira, SP, a maioria dos bananais amostrados apresenta deficiência de $\mathrm{N}$ e $\mathrm{S}$. A deficiência de $\mathrm{K}$ em alguns bananais pode ser atribuída ao desequilíbrio catiônico (K:Mg:Ca). O boro é o nutriente encontrado em excesso com maior frequência. A média do teor foliar de $\mathrm{K}, \mathrm{Ca}, \mathrm{S}, \mathrm{Cu}$ e Zn é maior no subgrupo Cavendish em relação ao Prata.

\section{REFERÊNCIAS}

1. BORGES, A. L. Interação entre nutrientes em bananeira. Cruz das Almas: Embrapa-CNPMF, 2004. 2 p. (EmbrapaCNPMF. Banana em Foco, 55)

2. CARPENA, R. M.; RITTER, A. R.; SOCORRO, N. PÉREZ. Nitrogen evolution and fate in a Canary Islands (Spain) sprinkler fertigated banana plot. Agricultural Water Management, v.52, n.2, p.93-117, 2002.

3. DAMATTO JUNIOR, E.R.; BOAS, R.L.V.; LEONEL, S.; FERNANDES, D.M. Avaliação nutricional em folhas de bananeira 'Prata-anã' adubadas com composto orgânico. Revista Brasileira de Fruticultura, v.28, n.1, p.109-112, 2006.

4. DONATO, S.L.R.; LEDO, A.A.; PEREIRA, M.C.T.; COELHO, E.F.; COTRIM, C.E; COELHO FILHO, M.A. Estado nutricional de bananeiras tipo Prata sob diferentes sistemas de irrigação. Pesquisa Agropecuária Brasileira, v.45, n.9, p. 980988,2010

5. FERREIRA, D. F. Análise estatística por meio do SISVAR (Sistema para Análise de Variância) para Windows versão 4.0. In: REUNIÂO ANUAL DA REGIÃO BRASILEIRA DA SOCIEDADE INTERNACIONAL DE BIOMETRIA, 45., 2000, São Carlos. Anais... São Carlos: UFSCar, 2000, p. 255-258.

6. FEY, R.; ZOZ, T.; STEINER, F.; RICHART, A.; BRITO, O. Lixiviação de nitrogênio em coluna em função da granulometria do solo. Scientia Agraria, v.11, n.2, p.181-185, 2010.

7. FURTADO, E. L.; BUENO, C. J.; OLIVEIRA, A. L.; MENTEN, J. O. M.; MALAVOLTA, E. Relações entre ocorrência do Mal -do-Panamá em bananeira da cv. Nanicão e nutrientes no solo e nas folhas. Tropical Plant Pathology, v.34 n.4, p. 211$215,2009$.

8. GODOY, L.J.G.; MENDONÇA, J.C.; OLIVEIRA, C.A.; GOMES, J.M. Fertilidade do solo em bananais do Vale do Ribeira. In.: GODOY, L.J.G.; GOMES, J.M (Ed.) Tópicos sobre a nutrição e adubação da cultura da banana. Registro: UNESP, 2009, p. 121-143.

9. GONÇALVEZ, J.S. et al. Bananicultura no Estado de São Paulo, Brasil. In: MORAES, W.S; OROZCO-SANTOS, M. OROSZO-ROMERO. Simpósio de manejo adequado da Sigatoka Negra na cultura da banana, 1., 2006 Pariquera-Açu. Anais... Pariquera-Açu: Pólo Regional da Apta Vale do Ribeira, 2006.

10. LÓPEZ, A.; ESPINOSA, J. Manual de nutricion y fertilizacion del banano. Quito: CORBANA, 1995. 82p.

11. MALAVOLTA, E.; VITTI, G.C.; OLIVEIRA, S.A. Avaliação do estado nutricional das plantas: princípios e aplicações. Piracicaba: POTAFOS, 1997. 201p.

12. MENDONCA, J.C.; PENTEADO, L.A.C.; GODOY, L.J.G. Nutrição e adubação da cultura da banana no Vale do Ribeira. In.: GODOY, L.J.G. (Ed.) Workshop sobre nutrição e adubação da cultura da banana. Pariquera-Açu: UNESP/APTA/ POTAFOS, 2006, p. 57-76.

13. MORAES, W.S.; LIMA, J.D. Nutrição mineral e sanidade da cultura da banana (Musa spp.). In.: GODOY, L.J.G. (Ed.) Workshop sobre nutrição e adubação da cultura da banana. Pariquera-Açu: UNESP/APTA/POTAFOS, 2006, p. 78102.

14. PRASERTSAK, P.; FRENEY, P.G.; DENMEAD, O.T.; PROVE, B.G. Fate of Urea Nitrogen Applied to a Banana Crop in the Wet Tropics of Queensland. Nutrient Cycling in Agroecosystems, v.59, n.1, p. 65-73, 2001.

15. RAIJ, B. van, CANTARELLA, H., QUAGGIO, J.A., FURLANI, A.M.C. (ed.) Recomendações de adubação e calagem para o Estado de São Paulo. Campinas: Instituto Agronômico/Fundação IAC, 1997. 285 p. (Boletim Técnico 100). 
GODOY, L.J.G. et al. Levantamento nutricional de bananais...

16. RODRIGUES, M.G.V.; RUGGIERO, C.; NATALE, W.; PACHECO, D.D. Nutrição e produção da bananeira 'Prata-anã' adubada com zinco e boro diretamente no rizoma, via muda desbastada. Revista Brasileira de Fruticultura, v.29, n.3, p. 645-651, 2007.

17. SILVA, E.B.; RODRIGUES, M.G.V. Levantamento nutricional dos bananais da região norte de Minas Gerais pela análise foliar. Revista Brasileira de Fruticultura, v. 23, n. 3, p. 695-698, 2001.

18. SILVA, J.T.A.; CARVALHO, J.G. Avaliação nutricional de bananeira 'Prata Anã' (AAB), sob irrigação no semi-árido do norte de Minas Gerais, pelo método DRIS. Ciência e Agrotecnologia, v.29, n.4, p.731-739, 2005.

19. SOARES, F.A.L.; GHEYI, H.R.; OLIVEIRA, F.H.T.; FERNANDES, P. D.; FRANCISCCO, A.N.A.; SILVA, F. V. Acúmulo, exportação e restituição de nutrientes pelas bananeiras "Prata Anã" e "Grand Naine, Ciência Rural, v.38, n.7, p.2054$2058,2008$.

20. SOUZA, J.S.; TORRES FILHO, P. Aspectos socioeconômicos. In: ALVES, E. J. (org.) A cultura da banana: aspectos técnicos, socioeconômicos e agroindustriais. 2 ed., Brasília: Embrapa-SPI / Cruz das Almas: Embrapa-CNPMF, 1999. $585 \mathrm{p}$

21. TEIXEIRA, L.A.J.; ZAMBROSI, F.C.B.; BETTIOL NETO, J.E. Avaliação do estado nutricional de bananeiras do subgrupo Cavendish no estado de São Paulo: normas DRIS e níveis críticos de nutrientes. Revista Brasileira de Fruticultura, v.29, n.3, p. 613-620, 2007.

22. VARGAS, A.; ARIAS, F.; SERRANO, E.; ARIAS M., O. Toxicidad de boro en plantaciones de banano (Musa AAA) en costa rica. Agronomía Costarricense, v.31,n.2, p.21-29, 2007.

Recebido em 13/04/2011

Aceito em 20/12/2011 